\title{
Low-dose Liver CT Images Segmentation and 3D Reconstruction
}

\author{
Fan Liu ${ }^{1, a}$, Xinyu Jinn ${ }^{1, b}$, Wenyuan Qiu ${ }^{1}$, Lanjuan $\mathrm{Li}^{2}$ \\ ${ }^{1}$ College of Information Science and Electronic Engineering, Zhejiang University, Hangzhou, 310027, \\ China \\ ${ }^{2}$ State Key Laboratory for Diagnosis and Treatment of Infectious Diseases, The First Affiliated \\ Hospital of Medical College , Zhejiang University, Hangzhou, 310027, China \\ aemail: flyingliufan@zju.edu.cn, bemail: jinworker@126.com
}

\begin{abstract}
Keywords: low-dose CT, liver, image segmentation, interpolation, 3D reconstruction.
Abstract. Computed tomography (CT) technology and three-dimensional (3D) reconstruction technology based on CT both play a significant part in many medical applications such as auxiliary diagnosis and surgery simulation [1]. But the radiation in CT may induce other diseases such as cancer. Therefore, low-dose CT becomes a research hot spot. In low-dose CT images, the random noise is more serious, and the spacing between adjacent CT images is also bigger. These features have brought challenges to the segmentation and 3D reconstruction in low-dose CT images. A new framework of segmentation and 3D reconstruction is proposed in this paper to process low-dose CT images. Then we conduct an experiment with low-dose liver CT images provided by a cooperation hospital in Zhejiang province, and the final results in segmentation and reconstruction are satisfactory.
\end{abstract}

\section{Introduction}

The traditional way to analyze CT images relies too much on doctor's experience and subjective judgment. However, with the increase in the amount of medical image data, it has become difficult for the traditional method to meet the need of current medical diagnosis. Through 3D reconstruction technology, the medical information such as the outline of organs can be displayed more realistic, and the efficiency and accuracy of clinical diagnosis can also be improved effectively.

$3 \mathrm{D}$ reconstruction of $\mathrm{CT}$ images is the process to extract 3D information from a series of two-dimensional (2D) CT images and display it in an intuitive way. 3D reconstruction of CT images mainly includes four parts: image preprocessing, image segmentation, image interpolation and 3D drawing. The performance of each part has an important influence on the final result of 3D reconstruction.

High-dose CT will cause high radiation damage, which may induce other diseases. So low-dose CT is widely used in many medical applications [2]. But in low-dose CT images, noise is more serious. Besides, the layer spacing of CT images is much bigger than the distance between the pixels, which may cause obvious terrace effect in the result of 3D reconstruction.

This paper uses the low-dose liver CT images of a cooperation hospital in Zhejiang province as experimental data, which are shown in figure 1 . Because of the limitation of low dose, from figure 1 we can see the resolution of the CT image is low, and the outline of liver is fuzzy. Besides, by analyzing the DICOM files, we know the layer spacing is 5 millimeter, which is much bigger than the pixel spacing.

\section{Preprocessing of CT Image}

The main task of preprocessing is de-noising. The current algorithm of image de-noising mainly includes the average filtering, Gaussian filtering, wiener filtering, anisotropic diffusion filter, etc. [3]. The main idea of the median filtering is to choose a suitable window in the center of the target pixel, of which the size of window is determined according to the actual application, and then to average the characteristic values of pixels within the window, and finally to use the average value as characteristic value of the target pixel. Similarly, the Gaussian filter is to obtain the weighted average 
within the window. These two methods can effectively suppress additive noise, but will lose edge details and cause image blur.

The liver boundary is fuzzy because of the low resolution ratio of low-dose CT image. So we should keep as much edge details of CT image as possible in the de-noising algorithm. Anisotropic diffusion filter de-noising method is based on partial differential equation [4]. This de-noising model determines the speed of diffusion according to the gradient of image, and can keep edge details at the same time when eliminating noise. In this paper, anisotropic diffusion filter is used to perform de-noising.

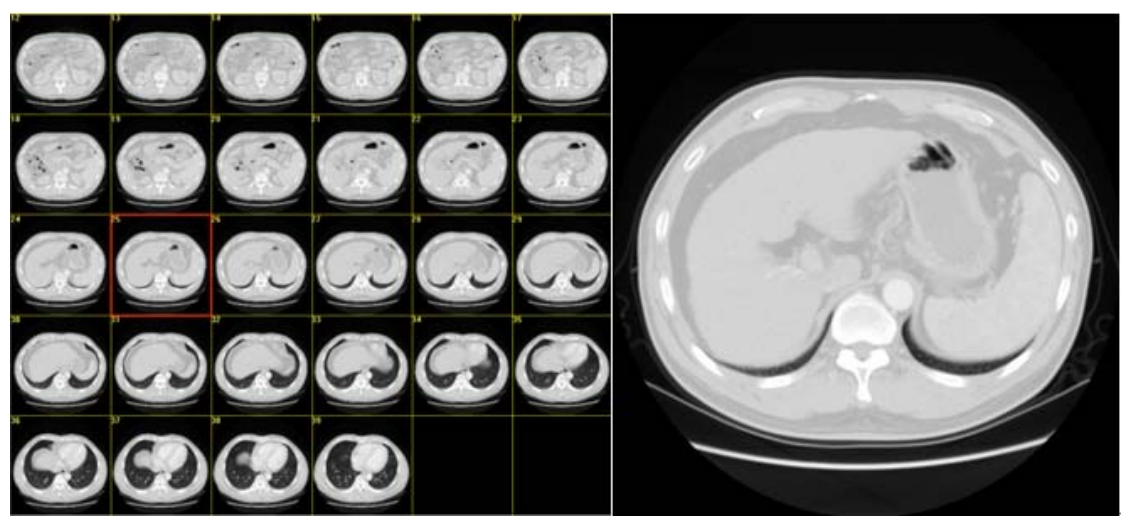

Fig.1. low-dose liver CT images

\section{D Segmentation of CT Image}

The difference between different kinds of medical image is so big that we have to choose an appropriate method according to the specific situation [5]. Medical image segmentation can be divided into two categories: 1) region based segmentation, methods of this kind usually use the uniformity in the same region to identify different regions of the image, including threshold method [6], region growing method [7], watershed method [8], etc. 2) edge based segmentation, methods of this kind often use the different properties between different regions to extract the dividing line between different regions. Liver CT image segmentation is to extract the liver region.

Level set is an edge based segmentation method. The algorithm uses zero contour of $\mathrm{N}$ dimension as the target boundary to segment goal of N-1 dimensional [9]. It has strong topological adaptability to deal with the large topological change of liver in CT images. So, level set segmentation algorithm is adopted in this paper. Figure 2 is the segmentation result of one liver CT image using this algorithm.

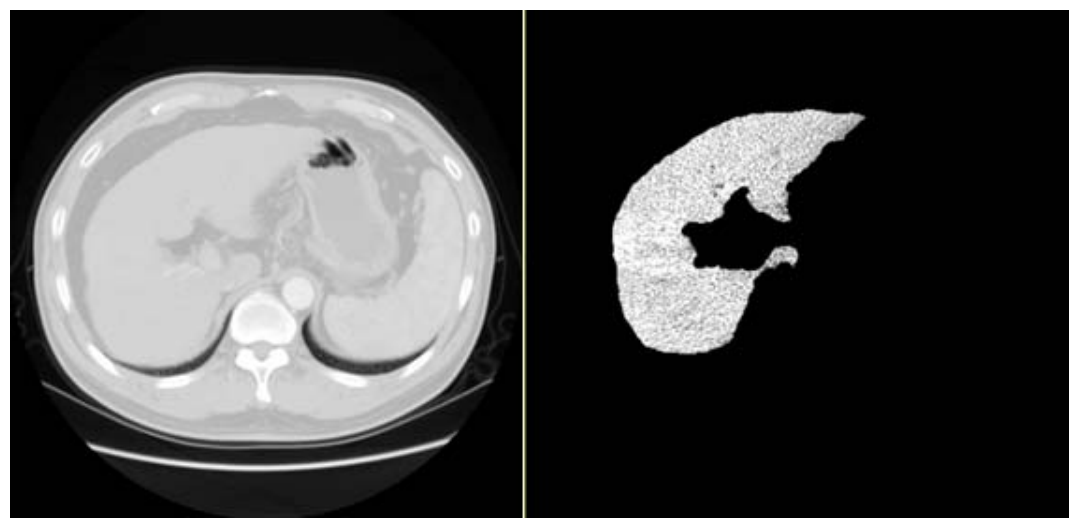

Fig.2. result of level set segmentation algorithm

\section{Low-dose CT Images Segmentation}

Because the layer spacing of low-dose CT image series is very large, we cannot generate accurate 3D data volume to use 3D segmentation algorithm directly. It is suitable to perform 3D segmentation through the combination of $2 \mathrm{D}$ segmentation of liver CT image. A semi-automatic segmentation 
framework based on 2D segmentation used to process the low-dose liver CT images is proposed in this paper, as shown in figure 3.

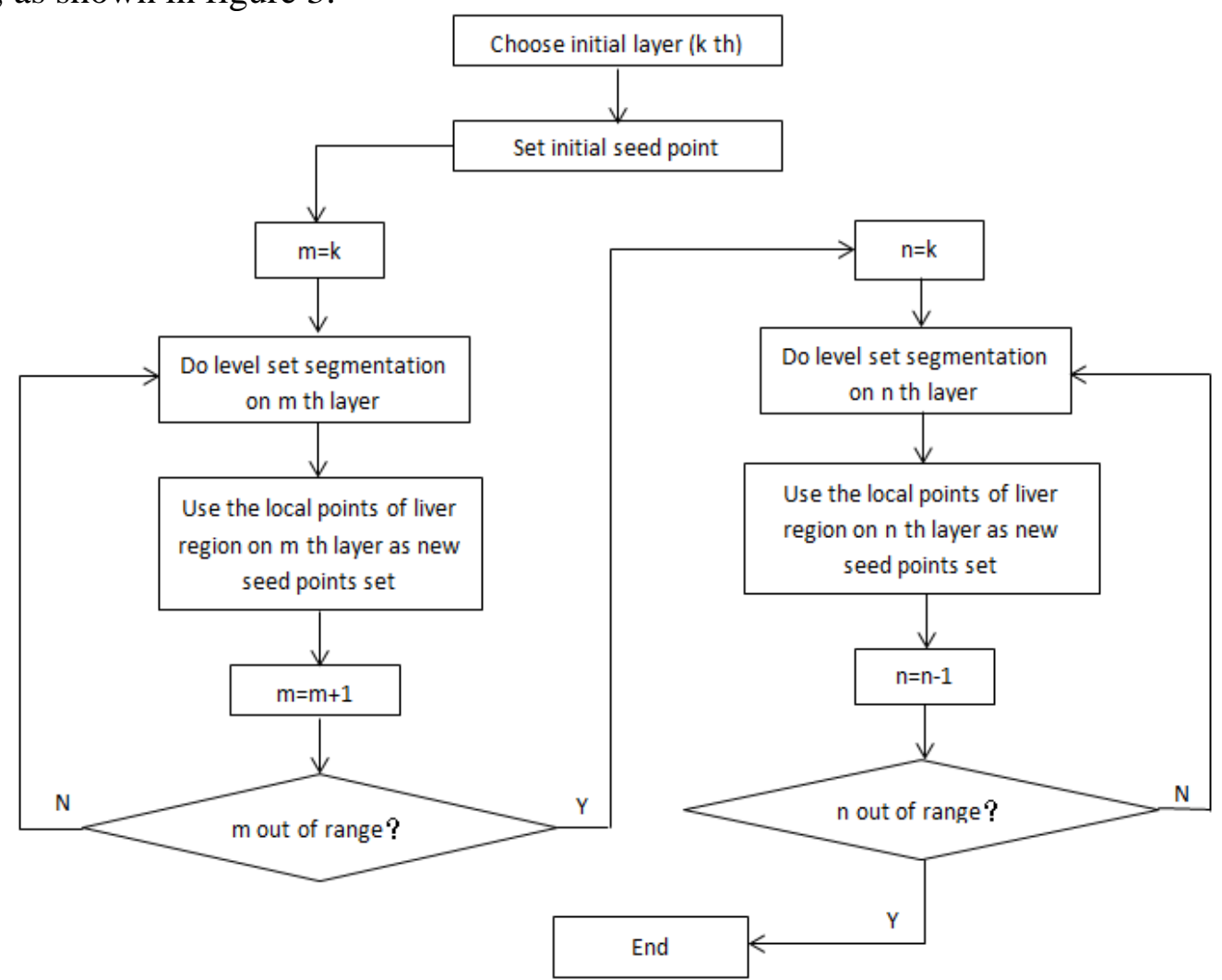

Fig.3. liver CT images segmentation framework

Firstly, we need select an initial CT image which has a clear and connected liver region, and specify a seed point within the liver region artificially. Then we use level set algorithm for the segmentation of the liver region according to the seed point. The result of segmentation is a liver region such as figure 2 . Now the problem is how to perform the segmentation of other CT images automatically. An algorithm based on distance transformation is proposed in this paper to realize this target.

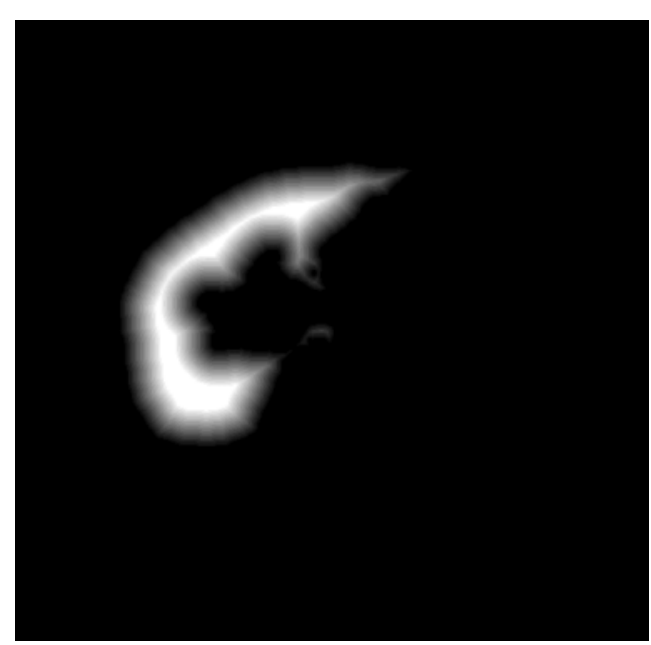

Fig.4. result of distance transformation

We fist transform the segmentation of initial CT image into a binary image, in which the value of liver region is 1 as target and the value of other region is 0 as background. Then distance transformation is performed on the binary image. The result of distance transformation is a gray level image, in which the value represents its minimum distance to background. Figure 4 is the result of distance transformation corresponding to the right part of figure 2. 
From figure 4 we can see that the points with local maximum grey value in distance graph can be taken as the local center of liver. Due to the coherence of liver region between adjacent liver CT image series, there is a large probability that one local center point of liver region on one CT image layer has a position in the liver region on adjacent CT image layer. So we can use the local center points of initial segmentation result as the seed points to perform level set segmentation to adjacent CT image layer. Similarly, we can use local center points of the new segmentation results as new seed points to do next level set segmentation, until the liver segmentation of all CT images is done. Using the segmentation framework proposed in this paper to the low-dose liver CT images, we can obtain segmentation result as shown in figure 5:

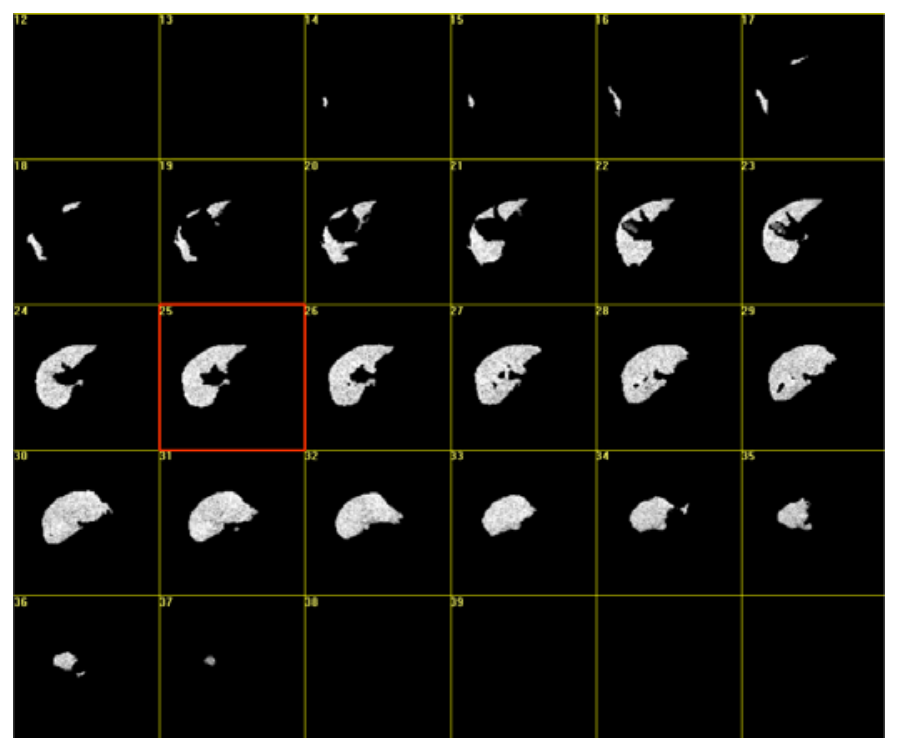

Fig.5. liver CT images segmentation result

\section{Interpolation and 3D Reconstruction}

When the segmentation result of liver CT images is obtained, if we perform 3D reconstruction on it directly, serious terrace effect will exist due to the lager layer spacing of low-dose CT images. Through interpolation between layers of the segmentation result, the severity of terrace effect can be reduced.

Image interpolation algorithm between layers can be divided into two categories: interpolation based on the gray value and interpolation based on shape. The main problem of gray value based interpolation methods is that the boundary of interpolation images is always fuzzy. Shape based interpolation can improve the problem, but it can only do interpolation to one region of interest.

Jian Wu proposed a kind of CT image interpolation algorithm based on contour shape, which has good performance on the interpolation of CT images [10]. But the algorithm has not considered small offset between two adjacent CT images due to reasons such as the human body movement. In this paper, we use image registration to improve the interpolation algorithm in [10]. After interpolation, we use volume rendering to obtain the final result of 3D reconstruction. The final result is shown in figure 6.
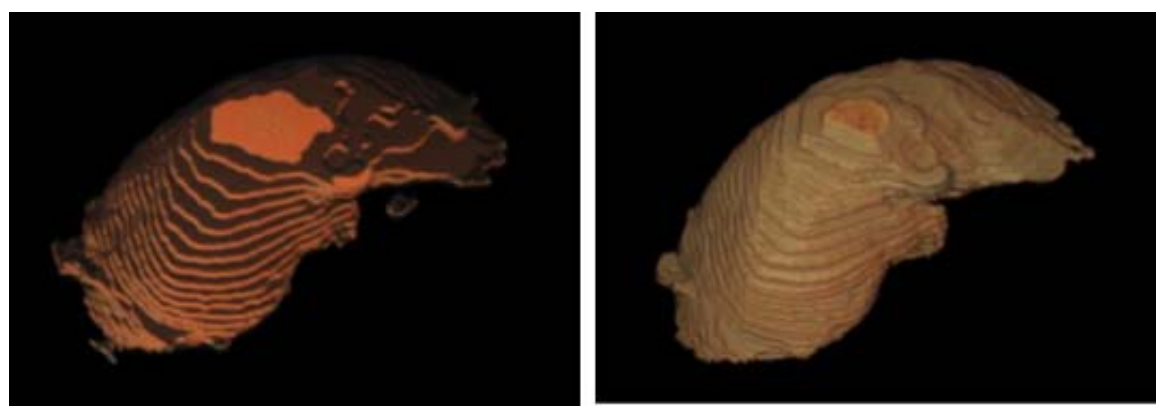

Fig.6. result of interpolation and 3D reconstruction 
In figure 6, the left image is the 3D reconstruction result without interpolation, in which the terrace effect is very obvious. The right image with better visual effect is the $3 \mathrm{D}$ reconstruction result after using the modified interpolation proposed in this paper.

\section{Conclusions}

Low-dose CT can effectively reduce the radiation damage, but the random noise in the image would be very serious, and the layer spacing is also large. In this paper, image processing algorithm, such as de-noising, segmentation and interpolation are analyzed and improved according to the characteristics of low-dose liver CT images. Besides, a semi-automatic segmentation framework suitable for low-dose CT images is proposed. Through experiment performed on real low-dose liver CT data, it is proved that the method proposed in this paper can successfully accomplish segmentation on the CT images, and obtain a satisfactory result of 3D reconstruction.

\section{Acknowledgements}

The paper's work is supported by the opening foundation of the State Key Laboratory for Diagnosis and Treatment of Infectious Diseases, the First Affiliated Hospital of Medical College, Zhejiang University, grant NO. 2014KF06, and National science and technology major projects, a new generation of broadband wireless mobile communication network NO.2013ZX03005013.

\section{References}

[1] Jie Tian, Shanglian Bao, Mingquan Zhou. Medical image processing and analysis[M]. Beijing: Electronic industry press, 2003. In Chinese.

[2] Lin Li, Dehong Luo. The Clinical Application of Low-dose CT Scanning[J]. Chinese Journal of Oncoradiology, Vol.2, No.4, p146-149, 2009. In Chinese.

[3] Buades A, Coll B, Morel J M. A review of image denoising algorithms, with a new one[J]. Multiscale Modeling \& Simulation, 2005, 4(2): 490-530.

[4] Weickert J. Anisotropic diffusion in image processing[M]. Stuttgart: Teubner, 1998.

[5] Wust P, Gellermann J, Beier J. Evaluation of segmentation algorithms for generation of patient models in radiofrequency hyperthermia[J].Phys. Med. Biol, 1998, 43: 3295-3307.

[6] Sahoo P K, S Soltani, A K C Wong. A survey of thresholding techniques[J]. Computer vision, graphics, and image processing, 1988. 41(2): 233-260.

[7] Hojjatoleslami S A, Kittler J. Region growing: a new approach[J]. IEEE Transactions on Image processing, 1998, 7(7): 1079-1084.

[8] Vincent L, Soille P. Watersheds in digital spaces: an efficient algorithm based on immersion simulations[J]. IEEE Transactions on Pattern Analysis \& Machine Intelligence, 1991 (6): 583-598.

[9] Tsai Y H R, Osher S. Total variation and level set methods in image science[J]. Acta Numerica, 2005, 14: 509-573.

[10] Jian Wu, Zhiming Cui, Feng Ye, Qun Wang. Interpolation based on contour shape for CT faulted images[J]. Computer Applications and Software, 2008, 25(11): 63-65. In Chinese. 